\title{
Ni@C Catalyzed Hydrogenation of Acetophenone to Phenylethanol under Industrial Mild Conditions in Flow Reactor
}

Shanshan Lin $^{\mathrm{a}, \mathrm{c}}$, Jianguo Liu ${ }^{\mathrm{b} *}$, Longlong $\mathrm{Ma}^{\mathrm{b} *}$

${ }^{a}$ CAS Key Laboratory of Renewable Energy, Guangdong Provincial Key Laboratory of New and Renewable Energy Research and Development, Guangzhou Institute of Energy Conversion, Chinese Academy of Sciences, Guangzhou 510640, P. R. China.

${ }^{\mathrm{b}}$ Key Laboratory of Energy Thermal Conversion and Control of Ministry of Education, School of Energy and Environment, Southeast University, Nanjing 210096, PR China.

c University of Chinese Academy of Sciences, Beijing 100049, P. R. China.

*Corresponding author: liujg@seu.edu.cn; mall@seu.edu.cn

\begin{abstract}
The catalytic hydrogenation of organic substrates containing many unsaturated functional groups is an important step in the industrial preparation of fine chemicals and has always been a hot spot in basic catalysis research. For example, the phenethyl alcohol obtained by the preferential hydrogenation of the $\mathrm{C}=\mathrm{O}$ group of acetophenone is a valuable intermediate for the production of spices, flavors, and medicines. Furthermore, as the demand for 1-phenylethanol $(\mathrm{PhE})$ continues to increase, the catalytic hydrogenation of acetophenone (AP) is becoming more and more important. The hydrogenation of acetophenone is a complex multi-step reaction. At present, relatively few catalytic systems are used in this reaction. The enantioselective hydrogenation on heterogeneous catalysts is due to its inherent operational and economic advantages, such as the atomic economy. It is one of the most ideal methods as it is easy to separate and recycle the catalyst. However, the traditional synthesis way in batch reactors usually takes a long time with an unsatisfying conversion which is not conducive to industrialization. Heterogeneous non-precious metal catalysts are advantageous for their implementation in flow reactor systems for industrial applications due to their ease of separation, low cost, and environmental protection. Herein, we report the first use of non-noble metal Ni-supported graphene-based catalysts for hydrogenation of acetophenone to phenylethanol with high efficiency in the flow reactor which can significantly improve mass and heat transfer. The conversion rate after optimizing the reaction conditions can be as high as $99.14 \%$ with a satisfactory conversion rate of $97.77 \%$. This catalyst is magnetic and has good cyclability. After 48 hours of uninterrupted continuous experiments, the Ni-based catalyst still maintains high catalytic activity, the conversion rate still reaches $88.44 \%$, and the catalyst structure remains intact and stable.
\end{abstract}

\section{KEYWORD}

Acetophenone; Hydrogenation; Phenylethanol; Ni@graphene catalyst; Flow reactor

\section{INTRODUCTION}

The chemical reduction of aromatic compounds (aromatic ketones, aromatic aldehydes, quinolines, etc.) through heterogeneous catalysis is an important field in the industrial hydrogenation process ${ }^{1}{ }^{2-10}$. As a valuable intermediate for the production of spices, 
edible flavors, and medicines, currently, the demand for the hydrogenated product of acetophenone, phenylethanol, has been large and increasing ${ }^{11-14}$. At present, most of the phenethyl alcohol in the industry is synthesized by chemical means using benzene or styrene. Its raw materials are all carcinogens, which are harmful to human health and the environment. In addition, the chemical synthesis of 1-phenylethanol often contains some by-products that are difficult to remove, which seriously affects the quality of the product. The synthesis of phenethyl alcohol by the reduction of unsaturated carbonyl compound acetophenone produces fewer by-products, and the synthesis route is simple, but because the reduction reaction usually requires a longer reaction time, it is not conducive to industrial production efficiency.

Generally, for the hydrogenation reaction, there are two hydrogenation technology methods: batch mode and flow reactor. In the batch reactor process, although high flexibility and high efficiency can usually be achieved under optimal conditions, gas/liquid/solid multiphase mixing is problematic, especially on large scales ${ }^{15}$. In addition, the $\mathrm{H}_{2}$ used in the hydrogenation process is a highly flammable gas that forms explosive mixtures with air in a wide range of concentrations. Therefore, in academic laboratories and industrial environments, handling the smallest and therefore the most penetrating gases is a big safety issue. Therefore, in order to avoid the inherent disadvantages of batch reactor methods, alternative flow reactor methods have been developed ${ }^{16}$. In this case, the flow reactor method means that two or more reagents are continuously pumped into the reactor to mix and react under precisely controlled conditions. This method has many advantages: accurate reaction parameters, good multi-phase mixing, automatic control, good reproducibility, and more importantly, high safety ${ }^{17}$ 18-21. The flow system can monitor and control the reaction process in real time, start or stop the reaction at any time, and can add raw materials during the reaction process, adjust the flow rate to control the reaction time as a flexible, controllable, convenient, and efficient reaction system. In particular, the implementation of continuous process practices in the pharmaceutical industry is considered to be one of the most strategically significant areas for innovation in green manufacturing methods ${ }^{22}$, 23. However, the use of flow reactors is not without challenges. In flow reactor hydrogenation, the currently used fixed catalysts may need to be significantly improved to provide good conversion efficiency and selectivity. In addition, solid blockage and multi-phase mixing in small-sized channels may affect or even completely stop the desired reaction. Therefore, the preparation of new catalysts and the optimization of flow conditions have become an indispensable challenge in the development of flow reactor hydrogenation.

Although there are many advanced and efficient precious metal-based catalysts for chemically hydrogenation ${ }^{10,24-29}$, the current trend in the pharmaceutical and food industries is the progress of economical, green, and environmentally friendly processes. Due to the high cost and scarcity of precious metals, it is very important and desirable to develop more cost-effective and practical application methods ${ }^{30,31}$. Through the development of new catalysts, such as metal catalysts, complex catalysts, and organic catalysts, the conversion efficiency and selectivity of hydrogenation have been continuously improved ${ }^{32}$. In order to improve the activity and recyclability of the 
catalyst, the researchers immobilized nano-scale catalytically active materials on various supports ${ }^{33-35}$. These heterogeneous catalysts show good robustness and have been used in industrial production including fine chemical manufacturing. As we all know, the development of non-precious metal heterogeneous catalysts has attracted much attention due to its obvious advantages such as low cost, comparable reactivity, separation and practicality, and recyclability ${ }^{36-38}$. The supported metal catalysts have attracted much attention due to their unique structure and better performance in certain catalytic reactions ${ }^{39,} 40$. In addition, Ni nanoparticles have been proved to be used for hydrogen transfer reduction of carbonyl compounds ${ }^{41-45}$.

Herein, we describe a simple and environmentally friendly method for the preparation of thin graphene spheres with a uniformly encapsulated Ni nano-alloy catalyst $(\mathrm{Ni} @ \mathrm{C})$ with nickel citrate as the precursor. The resulting catalysts are stable and reusable and have been successfully used in the hydrogenation reduction process of acetophenone to phenylethanol. This reaction is under very mild industrially feasible conditions $\left(100{ }^{\circ} \mathrm{C}\right.$ and $1 \mathrm{MPa} \mathrm{H}_{2}$ pressure), in a high-efficiency flow reactor system at a flow rate of $0.4 \mathrm{~mL} / \mathrm{min}$ to hydrogenate the easily available carbonyl compound acetophenone to phenethyl alcohol, which provides a feasible way of economical and efficient industrialization. In order to prevent the small catalyst particles from clogging the flow reactor pipeline, we adsorb the magnetic $\mathrm{Ni@C}$ catalyst on the magnetic stirring rod to leave room for liquid flow. Under optimized conditions, a continuous 48hour hydrogenation experiment of acetophenone was carried out in a flow reactor with non-toxic and cheap solvent ethanol, and the catalytic activity and product selectivity of the catalyst are not significantly reduced.

\section{EXPERIMENTAL SECTION}

\subsection{Materials}

1,3,5-trimethoxybenzene was purchased from Sigma Aldrich Co., Ltd.; $\mathrm{Ni}\left(\mathrm{NO}_{3}\right)_{2} \bullet 6$ $\mathrm{H}_{2} \mathrm{O}$ (AR, 98\%), anhydrous citric acid (AR, $\geq 99.5 \%$ ), commercial single ruthenium atom nitrogen-doped carbon catalyst was obtained from Shanghai Macklin Biochemical Co., Ltd.; $\mathrm{H}_{2} \mathrm{SO}_{4}$ (GR, 98\%) was purchased from Sinopharm Chemical Reagent Co., Ltd.; acetophenone (AR, $\geq 99.0 \%$ ), ethanol (AR, 99.7\%), commercial single palladium atom nitrogen-doped carbon catalyst, Raney nickel catalyst (20 40 meshes) were purchased from Aladdin (Shanghai) Chemical Technology Co., Ltd.; (+/-)1-phenylethanol (AR, 98.0\%) was purchased from Tokyo Chemical Industry Co., Ltd.; Deionized water $(\sigma<5 \mu \mathrm{S} / \mathrm{m})$ was self-made in the laboratory. Before using, the purity of acetophenone and phenylethanol has been checked.

\subsection{Preparation of $\mathrm{Ni} @ \mathrm{C}$ catalysts}

Nickel(II) nitrate hexahydrate $\left(\mathrm{Ni}\left(\mathrm{NO}_{3}\right)_{2} \cdot 6 \mathrm{H}_{2} \mathrm{O}, 0.03 \mathrm{~mol}\right)$ and citric acid $\left(\mathrm{C}_{6} \mathrm{H}_{8} \mathrm{O}_{7}\right.$, $0.03 \mathrm{~mol}$ ) were dissolved in $20 \mathrm{~mL}$ of absolute ethanol, and then the mixture was stirred at $70{ }^{\circ} \mathrm{C}(300 \mathrm{rpm})$ Aging for 4-8 hours until a bubble-like green gel is obtained. Place it in a drying oven at $100{ }^{\circ} \mathrm{C}$ for 24 to 72 hours to remove excess water. Then the green solid obtained was calcined in a fixed bed at $700{ }^{\circ} \mathrm{C}$ under $40 \mathrm{~mL} \mathrm{~min}^{-1}$ of high-purity 
$\mathrm{N}_{2}(99.999 \%)$ atmosphere for $3 \mathrm{~h}$, and the heating rate was controlled at $2{ }^{\circ} \mathrm{C} \min ^{-1}$. The resulting black solid was treated in a $1 \mathrm{M} \mathrm{H}_{2} \mathrm{SO}_{4}$ aqueous solution at $70{ }^{\circ} \mathrm{C}$ until the solution became colorless to remove unsafe and uncovered Ni particles. Then, the black solid was washed thoroughly with deionized water until the $\mathrm{pH}$ value of the waste liquid was 7. Finally, the black solid was dried in a freeze dryer at $-48^{\circ} \mathrm{C}$ for $12 \mathrm{~h}$ to obtain $\mathrm{Ni@C-700-EtOH}$ catalyst.

\subsection{Hydrogenation of acetophenone in a batch reactor}

The reaction was carried out in a stainless-steel autoclave (Shanghai Yanzheng Instrument Co., Ltd.), which had a $10 \mathrm{~mL}$ well, a thermocouple, and a circulating watercooling device. A glass lining was placed in the well, which contained a $10 \mathrm{~mm}$ magnetic stir bar, $0.5 \mathrm{mmol}$ acetophenone, $10 \mathrm{mg}$ catalyst, and $6 \mathrm{~mL}$ solvent. Then, the autoclave was sealed and purged with $\mathrm{H}_{2} 3$ times under a pressure of $1 \mathrm{MPa}$, and pressurized with the set target $\mathrm{H}_{2}$ pressure. Put the autoclave into the heating mantle and set the stirring speed to $300 \mathrm{rpm}$. The autoclave was preheated from room temperature to the target temperature (the internal temperature detected by the thermocouple) at a rate of $2^{\circ} \mathrm{C} \cdot \mathrm{min}^{-1}$. The target temperature was used as the reaction temperature. The reaction was carried out at the reaction temperature for the required time. After the reaction, the autoclave was cooled to room temperature and the remaining gas was discharged. The reaction solution was collected with a dropper and filtered. The catalyst was fixed on a magnetic stir bar and washed thoroughly with ethanol and water. Then used a freeze dryer to dry the catalyst (together with a magnetic stir bar) under vacuum at $-48{ }^{\circ} \mathrm{C}$ for 12 hours. The reaction product was identified by $\mathrm{GC}$, and the yield of the reaction product was determined by GC, using 1,3,5trimethoxybenzene as the internal standard.

\subsection{Hydrogenation of acetophenone in a flow reactor}

The reaction was carried out in a flow reactor (Ou Shisheng (Beijing) Technology Co., Ltd.). First, the flow reactor reaction tube was filled with magnets that could adsorb $100 \mathrm{mg}$ of catalyst, and the reaction tube was maintained at a specific reaction temperature and $\mathrm{H}_{2}$ pressure. Then, the acetophenone reaction raw material with a concentration of $0.083 \mathrm{~mol} / \mathrm{L}$ was replaced with gas. The mixed liquid was pumped into the reaction tube at a flow rate of $0.4 \mathrm{ml} / \mathrm{min}$, and the reacted mixed liquid flowed through the gas-liquid separator at a constant flow rate and entered the product collection device for gas phase and nuclear magnetic measurement. The reaction product was identified by GC and the yield of the reaction product was determined by GC, using 1,3,5-trimethoxybenzene as the internal standard.

\section{RESULTS AND DISCUSSION}

\subsection{Synthesis and characterizations of $\mathrm{Ni} @ \mathrm{C}$ catalysts}

These Ni@graphene catalysts are prepared using the improved simple synthesis method mentioned in section 2.2. The number of all prepared catalysts is M1/M2@C-a-b-c-d, where $\mathrm{M} 1=\mathrm{Ni} ; \mathrm{M} 2=\mathrm{NiO}$; $\mathrm{a}$ is the pyrolysis temperature; $\mathrm{b}$ is the oxidation temperature; $\mathrm{c}$ is the oxidation time and $\mathrm{d}$ is the solvent. Figure $\mathbf{1}$ shows representative scanning electron microscope (SEM) images of $\mathrm{Ni@C-700-EtOH}$ catalyst and $\mathrm{Ni@C-700-}$ EtOH(recycled) catalyst. As shown in Figures 1a and 1b, the surface of Ni@C-700- 
EtOH catalyst has an obvious pore structure, while the pore structure of Ni@C-700EtOH (recycling) catalyst deteriorates slightly after recovery but is still evenly distributed. Scanning electron microscope (SEM) image shows that the Ni@C-700EtOH sample (Figure 1a) has formed nanospheres with large pores with a uniform diameter of 600

$\mathrm{nm}$.

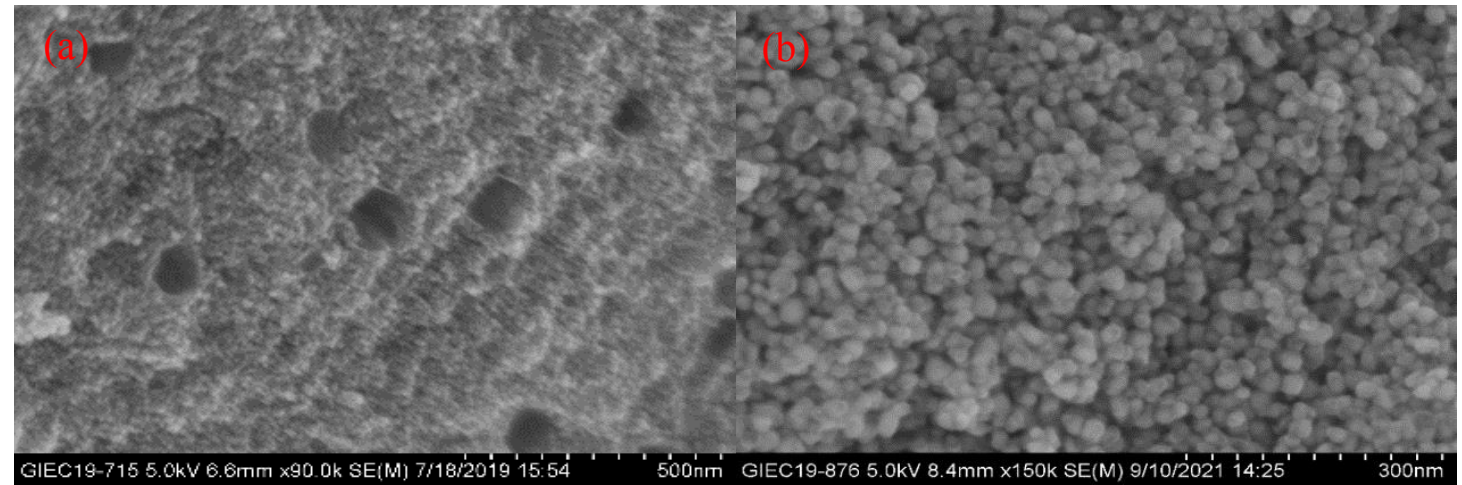

Figure 1. Representative SEM images of (a), Ni@C-700-EtOH (b), Ni@C-700-EtOH (recycled).
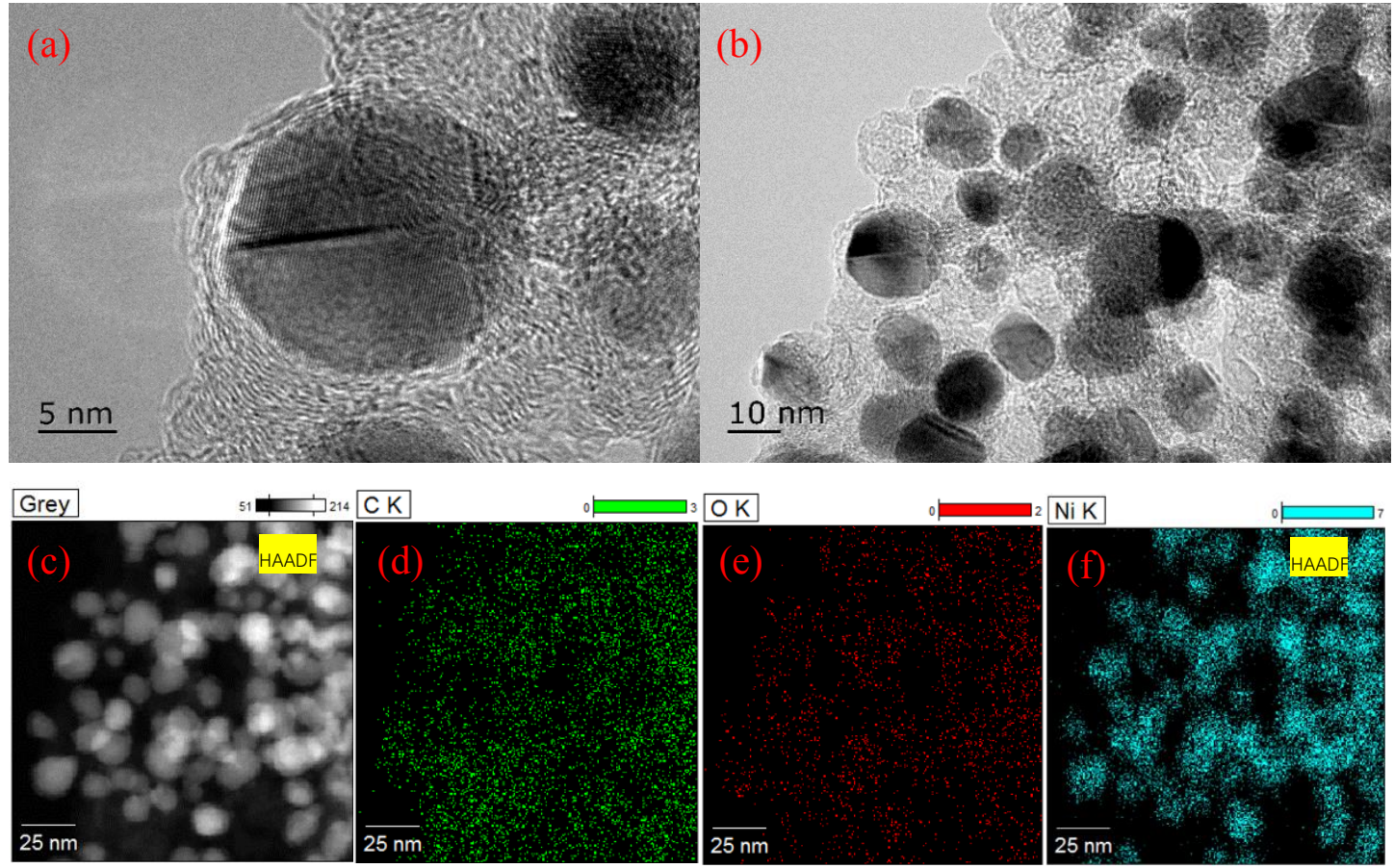

Figure 2. Catalyst characterization. HRTEM images of Ni@C-700-EtOH (a, b). HAADF-TEM images of a single particle in $\mathrm{Ni} @ \mathrm{C}-700-\mathrm{EtOH}$ (c) and corresponding EDS element mapping (EFTEM) of C, O, and HAADF-STEM analysis of $\mathrm{Ni}$ (d-f). 

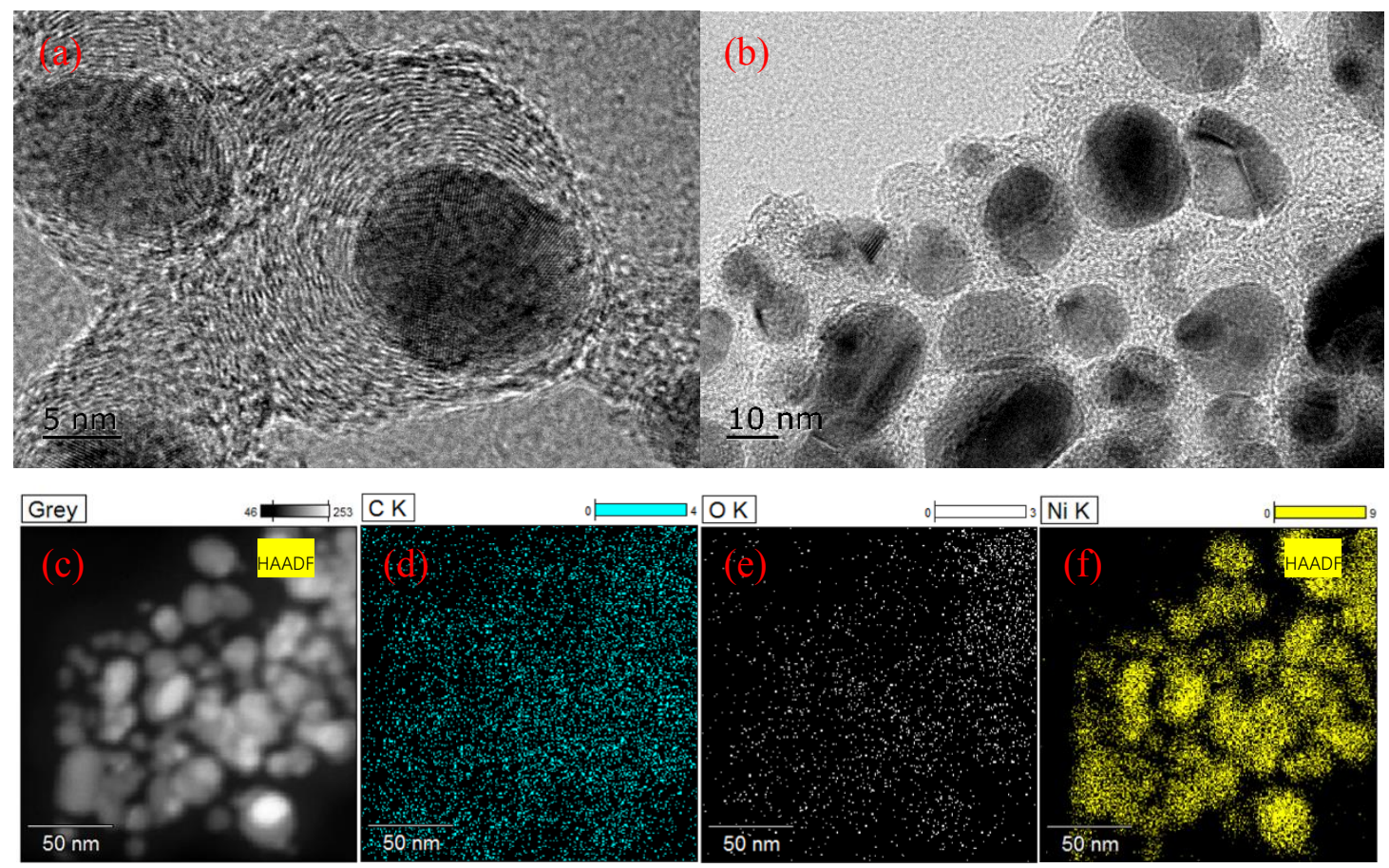

Figure 3. Catalyst characterization. HRTEM images of Ni@C-700-EtOH (recycled) (a, b). HAADF-TEM images of a single particle in Ni@C-700-EtOH (recycled) (c) and corresponding EDS element mapping (EFTEM) of C, O, and HAADF-STEM analysis of Ni (d-f).

Further high-resolution (HR) TEM analysis revealed that the nanospheres are composed of metal nanoparticles (NPs), which are completely coated with a graphene shell (Figure 2a and 2b). The Ni@C-700-EtOH metal NPs had a uniform size of generally 100-200 nm with a d-spacing of $0.20 \mathrm{~nm}$, which corresponds to the (111) plane of the Ni alloy (Figure 4c). For the Ni@C-700-EtOH (recycled) catalyst, the intensity of the weak peak $\mathrm{NiO}$ did not increase (Figure 4c), indicating that the reduction reaction process did not lead to the oxidation of the catalyst. Through statistical analysis of high-resolution transmission electron microscopy (HR-TEM), the graphene shells on nickel nanoparticles are very thin, and $>90 \%$ consists of a few layers. A high-angle annular dark-field scanning transmission electron microscopy (HAADFSTEM) image with sub-angstrom resolution further confirmed that uniform metal NPs had been formed (Figure 2c), and the corresponding energy-dispersive X-ray (EDX) maps showed that the Ni and $\mathrm{O}$ atoms were distributed homogeneously over all the NPs (Figure 2d-f). In addition, the EDX map of the recycled catalyst Ni@C-700-EtOH (recycled) (Figure 3d-f) showed no difference from the original catalyst which indicated that the reduction of acetophenone did not affect the distribution of $\mathrm{Ni}$ and $\mathrm{O}$ atoms on the NPs. The nitrogen physisorption measurements for the synthesized catalyst material in the specific surface area (Brunauer-Emmett-Teller method) were in accordance with the SEM phenomenon. Ni@C-700-EtOH has a surface area of 182 $\mathrm{m}^{2} \mathrm{~g}^{-1}$ and has a pore size distribution (> 95\% mesopores with a total pore volume of $0.269 \mathrm{~cm}^{3} \mathrm{~g}^{-1}$ and the average pore width of $5.6 \mathrm{~nm}$ ) (Figure $4 \mathbf{b}$ ). X-ray photoelectron spectroscopy (XPS) and X-ray diffraction (XRD) are used to study the electronic and structural properties of $\mathrm{Ni} @ \mathrm{C}-700-\mathrm{EtOH}$. XPS measurement indicated that both metallic $\mathrm{Ni}^{0}$ and $\mathrm{Ni}^{2+}$ species existed on the catalyst surface (Figure 4a). The XRD 
spectrum showed graphitic carbon shell C (002) between 20 and 30 which confirmed that a thin graphene shell has been formed. And it also showed that in addition to the graphite carbon shell and $\mathrm{Ni}$ alloy, there are also $\mathrm{NiO}$ phases in the samples (Figure 4c). This indicated that the active metal Ni species of the Ni@C catalysts prepared from the non-oxidation step were also oxidized by air after the reduction process during storage. These results indicate that the uniform nickel nano-alloy has been completely wrapped in the graphene thin shell.
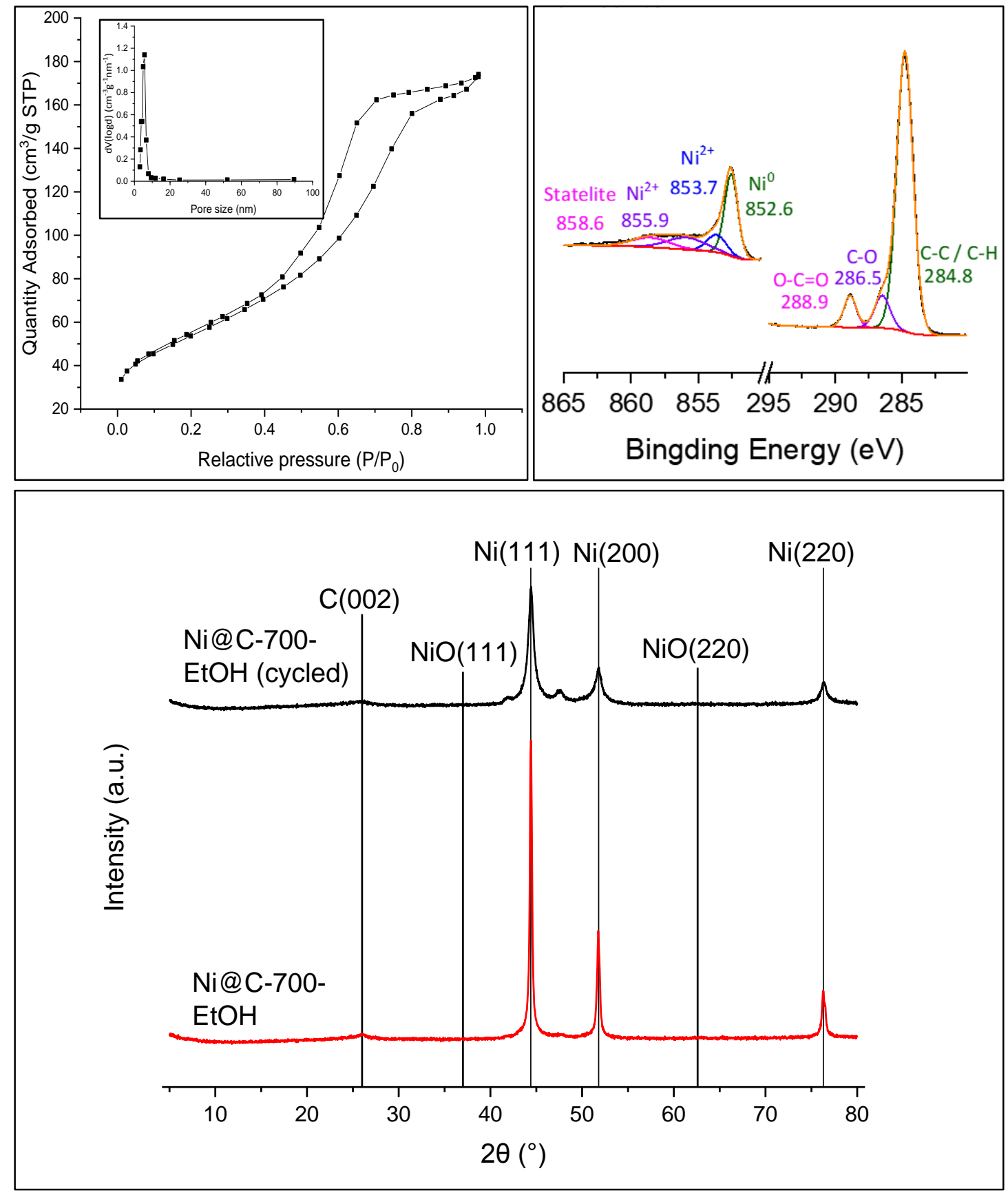

Figure 4. Catalyst characterization. BET measurements of $\mathrm{N}_{2}$ adsorption and desorption isotherm curves and pore size distribution profile of catalysts Ni@C-700-EtOH (a); XPS image of Ni@C-700-EtOH (b); XRD images of Ni@C-700-EtOH and Ni@C-700-EtOH (recycled) (c). 


\subsection{Hydrogenation of acetophenone using $\mathrm{Ni@C} \mathrm{catalysts} \mathrm{in} \mathrm{the} \mathrm{batch} \mathrm{reactor}$}

First, we tested all the prepared Ni@C catalysts and other commercially available catalysts $\mathbf{R u} / \mathbf{C}, \mathbf{P d} / \mathbf{C}$, and Raney nickel for the hydrogenation of acetophenone in a cheap, non-toxic ethanol solvent and $\mathrm{H}_{2}$ atmosphere for $15 \mathrm{~h}$. Figure 5 shows the distribution of phenethyl alcohol on catalysts with different yields and selectivities. As mentioned above, the oxidation step significantly affects the physical properties of the catalyst. Considering the comprehensive conversion rate and selectivity, the catalysts A, B prepared by the oxidation step perform better than the corresponding catalysts $\mathbf{D}$, E, $\mathbf{C}$ without the oxidation step. When the pyrolysis temperature of the unoxidized catalyst rises from 600 to 700 degrees Celsius, the catalyst activity has a significant decrease, while the catalyst activity after oxidation is less affected by the pyrolysis temperature. At a pyrolysis temperature of 700 degrees, the solvent used to prepare the catalyst also affects the catalytic activity and product selective activity. The catalyst $\mathbf{E}$ prepared from EtOH generally has worse reactivity and selectivity than the corresponding catalyst C, D prepared from $\mathrm{H}_{2} \mathrm{O}$. Among all Ni-based catalysts encapsulated by thin graphene layers, the catalyst F Ni/NiO@-700-200-1- $\mathrm{H}_{2} \mathrm{O}$ showed the best overall conversion and selectivity, which were $100 \%$ and $98.31 \%$, respectively. $\mathrm{Ni@C-700-EtOH}$ showed the worst reactivity. The catalytic activity of other commercially available noble metal catalysts $\mathbf{R u} / \mathbf{C}, \mathbf{P d} / \mathbf{C}$, and Raney nickel is not satisfactory. The noble metal catalysts $\mathbf{R u} / \mathbf{C}$ and $\mathbf{P d} / \mathbf{C}$ did not produce the target product, while Raney nickel showed good catalytic activity but with poor selectivity. In order to highlight the advantages of flow reactor over batch reactors, herein, we choose the prepared catalyst $\mathrm{Ni@C-700-EtOH}$ to conduct acetophenone hydrogenation experiments to screen the flow reactor process conditions. 


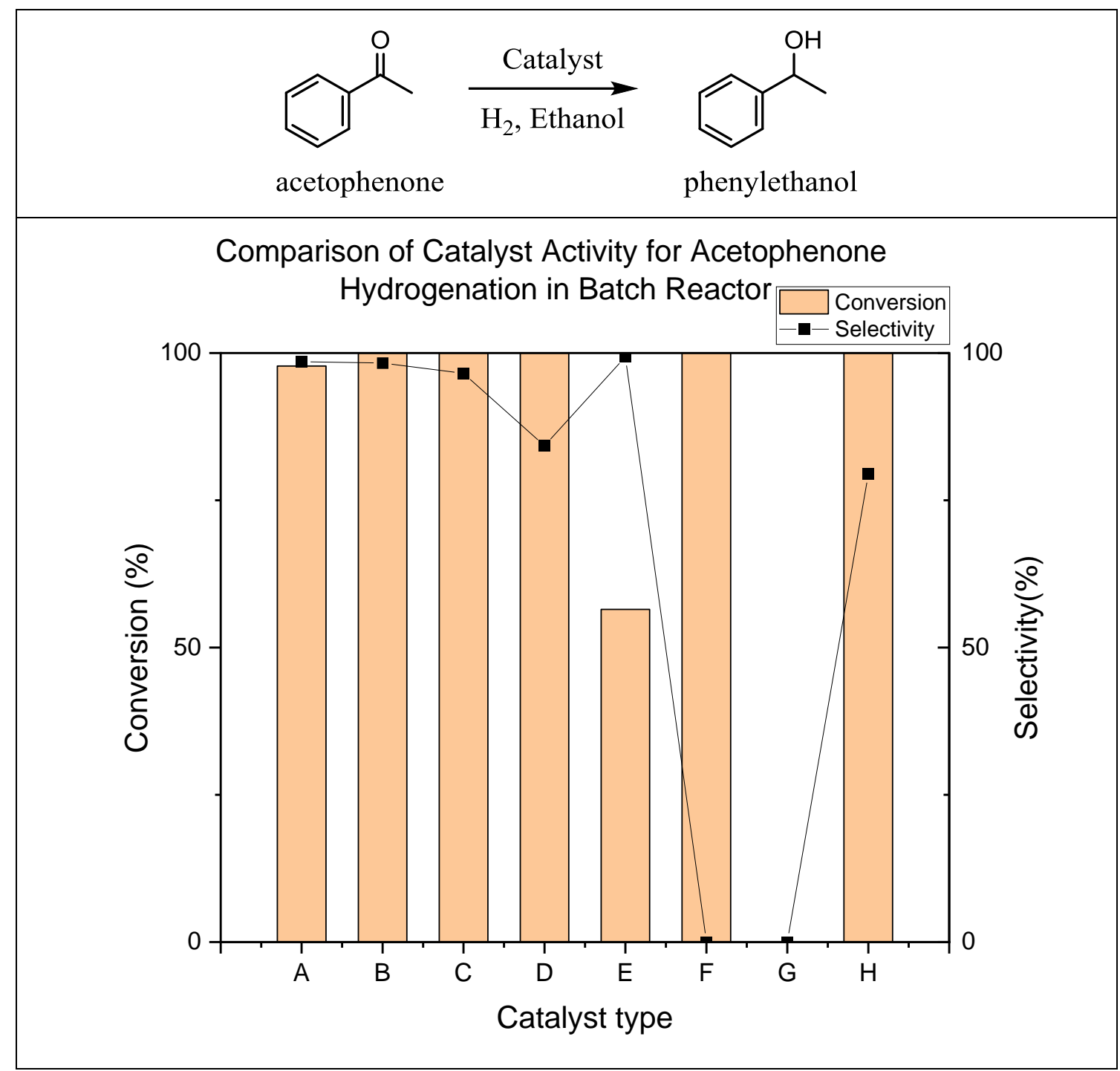

Figure 5. Catalyst Screening for the hydrogenation acetophenone. Reaction conditions: $10 \mathrm{mg}$ catalyst, $0.5 \mathrm{mmol}$ acetophenone, $6 \mathrm{~mL}$ Ethanol, $1 \mathrm{MPa} \mathrm{H}_{2}, 15$ h, $80^{\circ} \mathrm{C}$. A: Ni/NiO@C-600-200-1- $\mathrm{H}_{2} \mathrm{O}$, B: Ni/NiO@C-700-200-1$\mathrm{H}_{2} \mathrm{O}, \mathbf{C}: \mathrm{Ni} @ \mathrm{C}-600-\mathrm{H}_{2} \mathrm{O}, \mathbf{D}: \mathrm{Ni} @-700-\mathrm{EtOH}, \mathbf{E}: \mathrm{Ni} @ \mathrm{C}-700-\mathrm{EtOH}, \mathbf{F}: 5 \% \mathrm{Ru} / \mathrm{C}, \mathbf{J}: 5 \%$ Pd/C, H: Raney Nickel. Conversion and selectivity were determined by GC using 1, 3, 5-trimethoxybenzenen as an internal standard. The types of products were confirmed by GC

\subsection{Hydrogenation of acetophenone using Ni@C catalysts in the flow reactor}

Based on the above initial catalyst screening research, we used catalyst $\mathbf{E}$ to further test the reaction factors such as solvent, reaction temperature, hydrogen pressure, and reaction time. The schematic diagram of the $\mathrm{Ni} @ \mathrm{C}$ catalyst hydrogenation of acetophenone in a flow reactor is shown in Figure 6. 
(a)
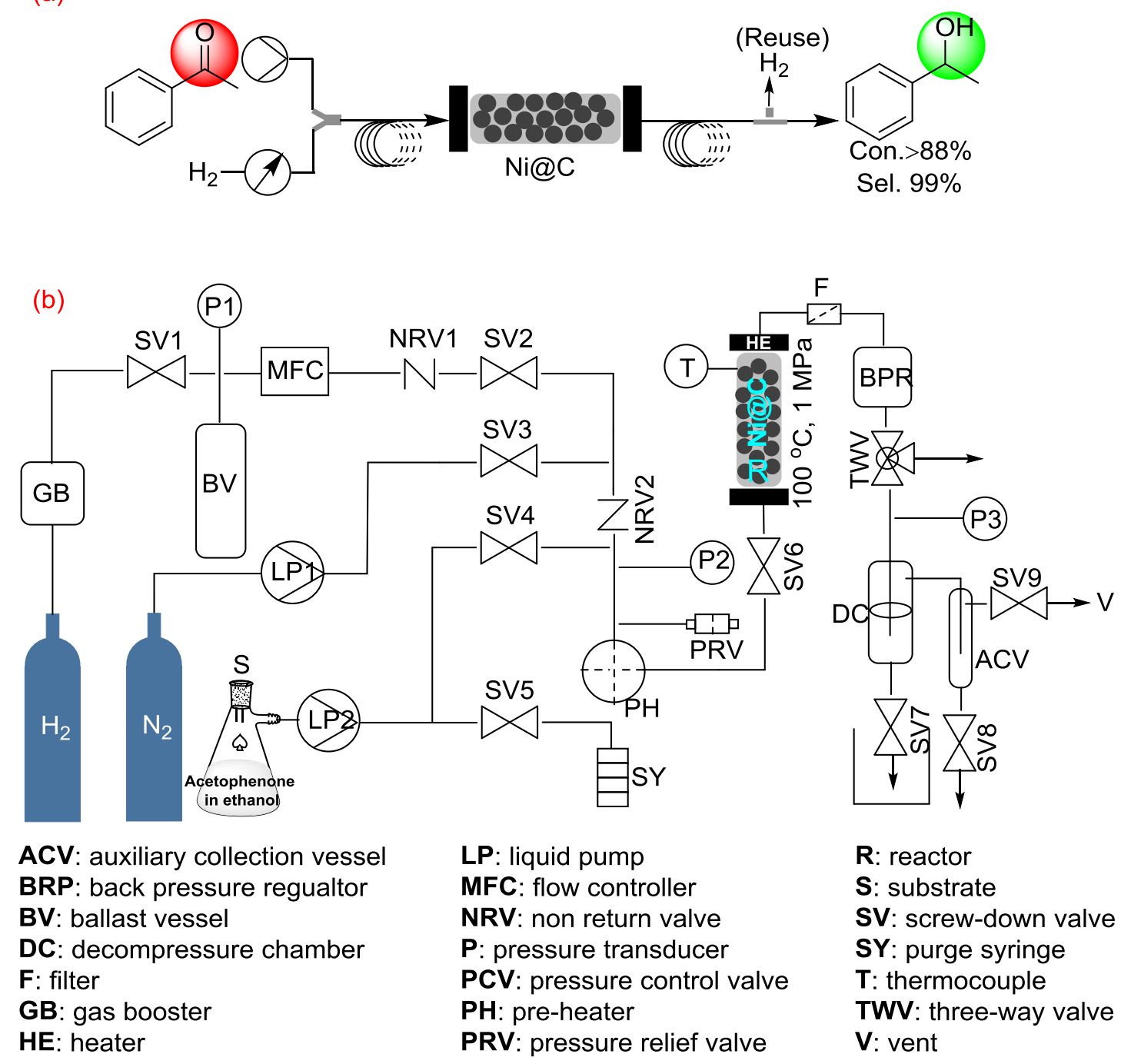

Figure 6. Schematic drawing for Ni@C catalyzed hydrogenation of acetophenone in flow reactor. Schematic diagram of the process of acetophenone in flow reactor (a); Schematic diagram of the pipeline structure of the flow reactor $(b)$.

Hydrogen pressure, as a key factor that usually significantly affects the hydrogenation reaction, is evaluated in the reaction of acetophenone hydrogenation to phenethyl alcohol at a reaction temperature of $120^{\circ} \mathrm{C}$ and a flow rate of $0.2 \mathrm{~mL} / \mathrm{min}$. As shown in Figure 7a, when the pressure increases from $2 \mathrm{MPa}$ to $2.5 \mathrm{MPa}$, the conversion rate and selectivity decrease to a certain extent. It is speculated that the pressure may be too high to vaporize acetophenone and float above the reaction tube, resulting in a smaller contact area with the catalyst. The catalytic activity is almost unaffected when the pressure is reduced from $2 \mathrm{MPa}$ to $1 \mathrm{MPa}$, only the selectivity changes slightly. When the pressure is $2 \mathrm{MPa}$, the selectivity is up to $99.9 \%$. When the pressure is reduced from $1 \mathrm{MPa}$ to $0.5 \mathrm{MPa}$, the catalytic activity of the catalyst drops sharply. In order to maintain the comprehensive conversion rate and selectivity, while making the reaction conditions as mild as possible for better application in industrialization, the pressure of $1 \mathrm{MPa}$ is selected for subsequent temperature and flow rate screening. As shown in Figure $\mathbf{7 b}$, in the temperature screening process, the catalyst reactivity is best when the temperature is $100^{\circ} \mathrm{C}$, and the selectivity is improved to a certain extent compared to $120^{\circ} \mathrm{C}$. During the temperature screening process, we found that the selectivity was the 
worst at the reaction temperature of $120^{\circ} \mathrm{C}$, indicating that the higher reaction temperature would lead to the formation of by-products. But when the temperature decreases furtherly, the conversion rate gradually drops significantly. Flow rate is also an important factor that affects industrial applications. We screened the flow rate under the selected reaction conditions of $1 \mathrm{MPa}$ and $100{ }^{\circ} \mathrm{C}$ as shown in the Figure 7c and found that appropriately increasing the flow rate can promote the selective production of phenylethanol. This indicated that short reaction time could not only reduce the production of by-products but also shortens the reaction time of the raw material acetophenone to the product phenethyl alcohol. Therefore, when the flow rate continues to increase from $0.4 \mathrm{~mL} / \mathrm{min}$ to $0.8 \mathrm{~mL} / \mathrm{min}$, the rate of decline of the conversion rate also increases. We used the optimized conditions to carry out the acetophenone hydrogenation reaction again in the batch reactor. The conversion rate after $15 \mathrm{~h}$ was only $15.04 \%$ relative to $99.14 \%$ in the flow reactor (Figure $7 \mathbf{d}$ ), indicating that the experiments we conducted in the flow reactor were very successful. Under the optimized reaction conditions, the 48-h continuous reaction of acetophenone without interruption was carried out, and phenylethanol with high selectivity remains basically unchanged, only the conversion rate is slightly reduced but still remains above $88.44 \%$.

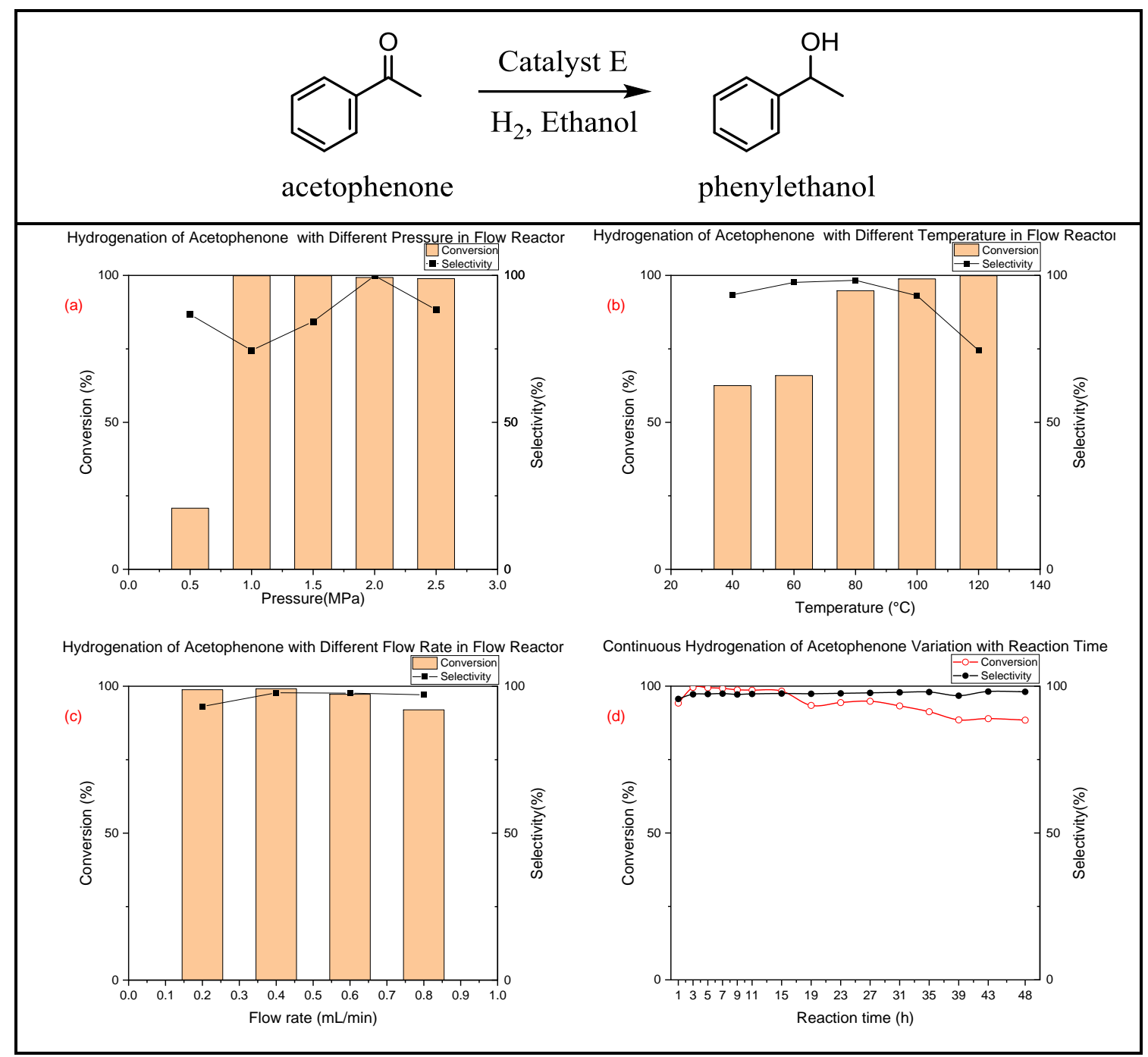

Figure 7. Study of reaction factors for the $\mathrm{Ni} @ \mathrm{C}$ catalyzed hydrogenation of acetophenone in flow reactor. Reaction conditions: (a) Reaction conditions: $10 \mathrm{mg}$ catalyst E Ni@C-700-EtOH, $0.08 \mathrm{~mol} / \mathrm{L}$ acetophenone (solvent: Ethanol), different $\mathrm{H}_{2}$ pressure, $120{ }^{\circ} \mathrm{C}, 0.2 \mathrm{~mL} / \mathrm{min}$; (b) Reaction conditions: $10 \mathrm{mg}$ catalyst $\mathbf{E} \mathrm{Ni} @ \mathrm{C}-700$-EtOH, 
$0.08 \mathrm{~mol} / \mathrm{L}$ acetophenone (solvent: Ethanol), $1 \mathrm{MPa} \mathrm{H}$, different reaction temperature, $0.2 \mathrm{~mL} / \mathrm{min}$; (c) Reaction conditions: $10 \mathrm{mg}$ catalyst E Ni@C-700-EtOH, $0.08 \mathrm{~mol} / \mathrm{L}$ acetophenone (solvent: Ethanol), $1 \mathrm{MPa} \mathrm{H}_{2}, 100^{\circ} \mathrm{C}, 0.2$ mL/min; (d) Reaction conditions: 10 mg catalyst E Ni@C-700-EtOH, 0.08 mol/L acetophenone (solvent: Ethanol), $1 \mathrm{MPa} \mathrm{H}_{2}, 100{ }^{\circ} \mathrm{C}$, different flow rate. Conversion and selectivity were determined by GC using 1, 3, 5trimethoxybenzenen as an internal standard.

\subsection{Proposed mechanism of graphene encapsulated Ni@C catalyzed hydrogenation of acetophenone}

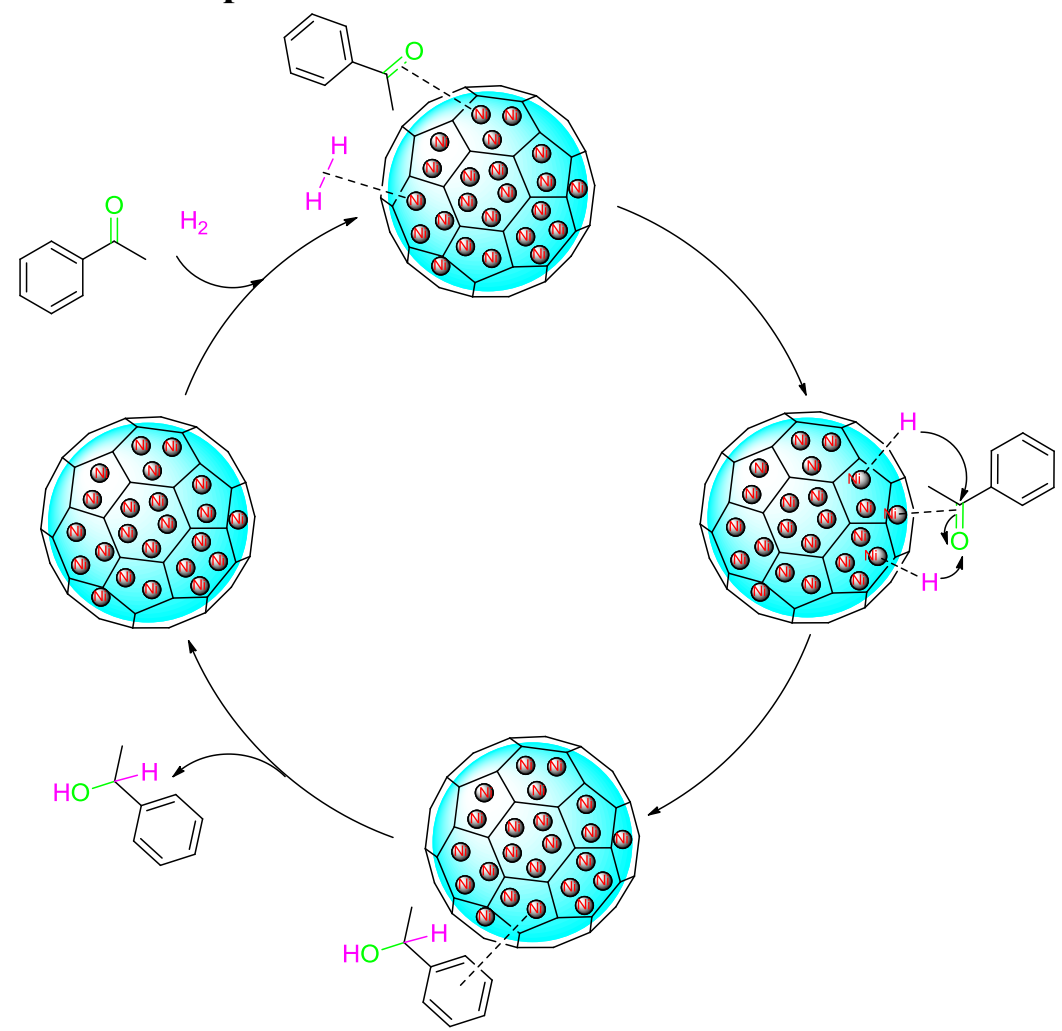

Figure 8. Proposed mechanism of graphene encapsulated Ni@C catalyzed hydrogenation of acetophenone The proposed mechanism of Ni-based catalyzed hydrogenation of ketones are showed in Figure 8. The hydrogen and ketone are both adsorbed on the surface of the Ni metal catalyst. Then a molecule of $\mathrm{H}_{2}$ which binds to the Ni active surface dissociates the $\mathrm{H}-\mathrm{H}$ bonds and forms $\mathrm{Ni}-\mathrm{H}$ (nickel hydride bonds) coordinate complex that is now ready to react with carbon-oxygen double bond of the acetophenone. In the meantime, a hydrogen in the metal-hydrogen complex is transferred to oxygen which involves a oxygen-metal bond. In the next step, the nickel-oxygen bond is broken and the second carbon-hydrogen bond is formed. After that, the desired product is desorbed from the catalyst surface.

\section{CONCLUSION.}

In conclusion, we have developed a highly efficient magnetic catalyst graphene encapsulated thin layer $\mathrm{Ni@C} \mathrm{for} \mathrm{highly} \mathrm{active} \mathrm{hydrogenation} \mathrm{of} \mathrm{acetophenone} \mathrm{to}$ phenylethanol and successfully applied it in a flow reactor with an excellent conversion rate and selectivity. The oxidation step, pyrolysis temperature, and solvent have an impact on the catalytic activity and product selectivity of the catalysis. We selected $\mathrm{Ni@C-700-EtOH}$ with the lowest hydrogenation activity of acetophenone among the 
prepared catalysts in batch reactor to carry out the hydrogenation reaction in the continuous flow reactor to highlight the advantages of the flow reactor which can significantly improve mass and heat transfer. Hydrogen pressure, reaction temperature, and reaction liquid flow rate have significant effects on the hydrogenation conversion rate and selectivity. In the final screening and optimization of the industrially adapted reaction conditions, the continuous hydrogenation of acetophenone was carried out for 48 hours to test the recyclicity of the catalyst. After 48 hours, the conversion rate and selectivity remained high. Compared with batch reactors, under optimized conditions, the catalytic activity of acetophenone hydrogenation in continuous flow reactor has been greatly improved. In short, the active catalyst loaded with a graphene shell has excellent stability which can be successfully used in advanced industrially applicable flow reactor by simply switching different control valves for the hydrogenation of acetophenone to benzene phenylethanol with excellent yields.

\section{ACKNOWLEDGMENT}

This work was supported financially by the National Key R\&D Program of China (2018YFB1501500), National Natural Science Foundation of China (Project 51976225).

\section{AUTHOR CONTRIBUTIONS.}

J. G. L. and L. L. M supervised and designed the research. S.S.L. performed the experiments and data analysis. S.S.L. and J. G. L. co-wrote the original manuscript and prepared the supporting information. J. G. L. corrected the original manuscript and supporting information. All authors discussed the results and assisted during manuscript preparation.

\section{COMPETING INTERESTS.}

The authors declare no competing financial interests.

\section{DATA ABAILABILITY}

Data supporting the findings of this study are available from the corresponding authors upon reasonable request.

\section{REFERENCES.}

1 E. T. Silveira, et al., The partial hydrogenation of benzene to cyclohexene by nanoscale ruthenium catalysts in imidazolium ionic liquids, Chem. Eur. J., 2004, 10, 3734-3740.

2 F. Jutz, et al., A green pathway for hydrogenations on ionic liquid-stabilized nanoparticles, $\mathrm{J}$ Catal, 2009, 268, 356-366.

3 J. Julis, et al., Selective hydrogenation of biomass derived substrates using ionic liquidstabilized ruthenium nanoparticles. Green Chem., 2010, 12 , 1634-1639.

4 D. Gonzalez-Galvez, et al., NHC-stabilized ruthenium nanoparticles as new catalysts for the hydrogenation of aromatics, Catal. Sci. Technol., 2013, 3, 99-105.

5 D. M. Zhu, et al., Aqueous Phase Hydrogenation of Quinoline to Decahydroquinoline Catalyzed 
by Ruthenium Nanoparticles Supported on Glucose-Derived Carbon Spheres, Chemcatchem, 2014, 6, 2954-2960.

$6 \quad$ L. Zhang, et al., Cooperation between the surface hydroxyl groups of $\mathrm{Ru}^{-} \mathrm{SiO}_{2} @ \mathrm{mSiO}(2)$ and water for good catalytic performance for hydrogenation of quinoline, Catal. Sci. Technol., 2014, 4, 1939-1948.

7 Y. P. Sun, et al., Complete hydrogenation of quinoline over hydroxyapatite supported ruthenium catalyst, Catal. Commun., 2010, 12, 188-192.

8 G. S. Fonseca, et al., Iridium nanoparticles prepared in ionic liquids: An efficient catalytic system for the hydrogenation of ketones, Synlett, 2004, 1525-1528.

9 V. Kogan, et al., Preferential catalytic hydrogenation of aromatic compounds versus ketones with a palladium substituted polyoxometalate as pre-catalyst, New J. Chem., 2002, 26, 272-274.

C. S. Chen, et al., Study of selective hydrogenation of acetophenone on $\mathrm{Pt} / \mathrm{SiO}_{2}$, Appl. Catal. AGen., 2003, 248, 117-128.

L. Cheng, et al., Effect of Temperature on B-Phenylethanol Anabolism in Saccharomyces Cerevisiae and its Recombinant Strains, Rom. Biotech. Lett., 2016, 21, 11550-11559.

C. Y. Cheng, et al., Enantioselective synthesis of S-(-)-1-phenylethanol in Candida utilis semifed-batch cultures, Process Biochem., 1996, 31, 119-124.

K. D. Kim, et al., The Comparative Effect of Particle Size and Support Acidity on Hydrogenation of Aromatic Ketones, Chemcatchem, 2019, 11, 4810-4817.

S. R. More, et al., Effect of Supercritical $\mathrm{CO}_{2}$ as Reaction Medium for Selective Hydrogenation of Acetophenone to 1-Phenylethanol, ACS Omega, 2018, 3, 7124-7132.

M. B. Widegren, et al., Towards practical earth abundant reduction catalysis: design of improved catalysts for manganese catalysed hydrogenation, Catal. Sci. Technol., 2019, 9, 6047-6058.

A. I. Stankiewicz, et al., Process intensification: Transforming chemical engineering (vol 96, pg 22, 2000), Chem. Eng. Prog. 2000, 96, 8-8.

J. Yoshida, et al., Flash chemistry: flow chemistry that cannot be done in batch, Chem. Commun., 2013, 49, 9896-9904.

C. Wiles, et al., Continuous flow reactors: a perspective, Green Chem., 2012, 14, 38-54.

S. V. Ley, et al., New tools and concepts for modern organic synthesis, Nat. Rev. Drug Discov., 2002, 1, 573-586.

K. Geyer, et al., Microreactors as tools for synthetic chemists - The chemists' round-bottomed flask of the 21st century? Chem. Eur. J., 2006, 12, 8434-8442.

R. L. Hartman, et al., Deciding Whether To Go with the Flow: Evaluating the Merits of Flow Reactors for Synthesis, Angew. Chem. Int. Ed., 2011, 50, 7502-7519.

22 S. G. Newman, et al., The role of flow in green chemistry and engineering, Green Chem., 2013, $15,1456-1472$.

C. Wiles, et al., Continuous process technology: a tool for sustainable production, Green Chem., 2014, 16, 55-62.

M. Casagrande, et al., Liquid phase acetophenone hydrogenation on $\mathrm{Ru} / \mathrm{Cr} / \mathrm{B}$ catalysts supported on silica, J. Mol. Catal. A Chem., 2002, 188, 133-139.

L. Cerveny, et al., Catalytic hydrogenation of aromatic aldehydes and ketones over ruthenium catalysts, Res. Chem. Intermed., 1996, 22, 15-22.

26 M. A. Aramendia, et al., Reduction of Acetophenones over Pd Alpo4 Catalysts - Linear FreeEnergy Relationship (Lfer), J. Catal., 1993, 140, 335-343. 
A. Drelinkiewicza, et al., Acetophenone hydrogenation on polymer-palladium catalysts, The effect of polymer matrix. Catal. Lett., 2004, 94, 143-156.

28 G. F. Santori, et al., Hydrogenation of aromatic ketones with Pt- and Sn-modified Pt catalysts, Appl. Catal. A-Gen., 2004, 269, 215-223.

29 M. M. Chen, et al., Hydrogenation of Acetophenone on Pd/Silica-Alumina Catalysts with Tunable Acidity: Mechanistic Insight by In Situ ATR-IR Spectroscopy, ACS Catal., 2018, 8, 6594-6600.

30 T. Yasukawa, et al., Chiral metal nanoparticle-catalyzed asymmetric C-C bond formation reactions, Chem. Soc. Rev. 2014, 43, 1450-1461.

31 S. Schimpf, et al., Supported gold nanoparticles: in-depth catalyst characterization and application in hydrogenation and oxidation reactions, Catal. Today 2002, 72, 63-78.

X. C. Lan, Highly Selective Catalysts for the Hydrogenation of Unsaturated Aldehydes: A Review, ACS Catal., 2020, 10, 2764-2790.

J. C. Pastre, et al., Flow chemistry syntheses of natural products, Chem. Soc. Rev., 2013, 42, 8849-8869.

34 M. Schreier, et al., Covalent Immobilization of a Molecular Catalyst on $\mathrm{Cu}_{2} \mathrm{O}$ Photocathodes for $\mathrm{CO}_{2}$ Reduction. J. Am. Chem. Soc., 2016, 138, 1938-1946.

A. Das, er al., Noncovalent Immobilization of Molecular Electrocatalysts for Chemical Synthesis: Efficient Electrochemical Alcohol Oxidation with a Pyrene-TEMPO Conjugate, Angew. Chem. Int. Ed., 2017, 56, 8892-8897.

A. F. Trasarti, et al., Liquid-phase hydrogenation of acetophenone over silica-supported Ni, Co and $\mathrm{Cu}$ catalysts: Influence of metal and solvent, Appl. Catal. A-Gen., 2014, 475, 282-291.

N. M. Bertero, et al., Catalytic and kinetic study of the liquid-phase hydrogenation of acetophenone over $\mathrm{Cu} / \mathrm{SiO}_{2}$ catalyst, Appl. Catal. A-Gen., 2008, 349, 100-109.

F. Zaccheria, et al., Heterogeneous selective catalytic hydrogenation of aryl ketones to alcohols without additives, Tetrahedron Lett., 2005, 46, 3695-3697.

39 R. G. Chaudhuri, et al., Core/Shell Nanoparticles: Classes, Properties, Synthesis Mechanisms, Characterization, and Applications, Chem. Rev., 2012, 112, 2373-2433.

L. Zhang, et al., Fabrication of $\mathrm{Fe}_{3} \mathrm{O}_{4} / \mathrm{PAH} / \mathrm{PSS} @$ Pd core-shell microspheres by layer-by-layer assembly and application in catalysis, J. Colloid Interface Sci., 2014, 421, 1-5.

F. Alonso, et al., Hydrogen-transfer reduction of carbonyl compounds catalysed by nickel nanoparticles, Tetrahedron Lett., 2008, 49, 1939-1942.

F. Alonso, et al., Hydrogen-transfer reduction of carbonyl compounds promoted by nickel nanoparticles, Tetrahedron 2008, 64, 1847-1852.

43 M. V. Rajashekharam, et al., Hydrogenation of acetophenone using a 10\% Ni supported on zeolite Y catalyst: kinetics and reaction mechanism, Catal. Today, 1999, 48, 83-92.

R. V. Malyala, et al., Activity, selectivity and stability of Ni and bimetallic Ni-Pt supported on zeolite Y catalysts for hydrogenation of acetophenone and its substituted derivatives, Appl. Catal. A-Gen., 2000, 193, 71-86.

45 N. M. Bertero, et al., Solvent effect in the liquid-phase hydrogenation of acetophenone over $\mathrm{Ni} / \mathrm{SiO}_{2}$ : A comprehensive study of the phenomenon, Appl. Catal. A-Gen., 2011, 394, 228-238. 